



\section{Lectio Magistralis}

ISSN 2612-7725 (PRINT) | ISSN 2704-5935 (ONLINE)

$-19-$ 
Comitato SCIENTIFICO

\section{Lezioni e Letture della Scuola di Scienze politiche "Cesare Alfieri"}

\author{
Direttore \\ Giusto Puccini \\ Presidente della Scuola di Scienze politiche "Cesare Alfieri" \\ Comitato Scientifico \\ Franca Alacevich \\ Professore di Sociologia economica \\ Giuseppe Coco \\ Professore di Economia politica \\ Carlo Fusaro \\ Professore di Diritto pubblico comparato \\ Massimo Morisi \\ Professore di Scienza politica \\ SANDRo Rogari \\ Professore di Storia contemporanea
}

Titoli

PUBBLICATI

\section{Inaugurazione anno accademico}

Sandro Rogari, Nazione e Stato nella storia d'Italia, 2010

Alessandro Cigno, Debito pubblico, ricchezza privata e politiche per la famiglia, 2012

Giusto Puccini, Rendimento e attualità della Costituzione repubblicana, 2014

Sergio Caruso, Per una nuova filosofia della cittadinanza, 2014 Carlo Fusaro, Rappresentare e governare: da grande regola a tallone d'Achille del governo parlamentare, 2015

Massimo Morisi, Tra regole ed eccezioni. La messa in opera delle politiche pubbliche in Italia, 2016

Carlo Trigilia, Crescita economica e coesione sociale nelle democrazie avanzate. Un divorzio inevitabile?, 2017

Gustavo De Santis, Il sistema previdenziale pubblico tra vincoli e scelte, 2019

\section{Lettura "Cesare Alfieri"}

Gustavo Zagrebelsky, La difficile democrazia, 2010

Colin Crouch, Europe and problems of marketization: from Polanyi to Scharpf, 2013

Marino Regini, La riforma universitaria nel quadro dei sistemi di governance europei, 2014

Nicoletta Maraschio, La lingua italiana tra passato e futuro, 2015 Jürgen Kocka, Capitalism is not democratic and democracy not capitalistic. Tensions and opportunities in historical perspective, 2015 Giuliano Amato, Le identità nazionali nell'Unione Europea, 2018 Marc Lazar, I mutamenti delle democrazie contemporanee. I casi della Francia e dell'Italia, 2019

Franca Alacevich, Senza lavoro? Cambiamento tecnologico e nuove sfide per la società, 2019 
Franca Alacevich

\section{Senza lavoro? \\ Cambiamento tecnologico e nuove sfide per la società}

Firenze University Press 
Senza lavoro? : cambiamento tecnologico e nuove sfide per la società / Franca Alacevich. - Firenze : Firenze University Press, 2019.

(Lectio Magistralis ; 19)

https://www.fupress.com/isbn/9788855180061

ISSN 2612-7725 (print)

ISSN 2704-5935 (online)

ISBN 978-88-5518-004-7 (print)

ISBN 978-88-5518-006-1 (online PDF)

ISBN 978-88-5518-008-5 (online EPUB)

Progetto grafico di Alberto Pizarro Fernández, Lettera Meccanica SRLs

\section{Certificazione scientifica delle Opere}

Tutti i volumi pubblicati sono soggetti a un processo di referaggio esterno di cui sono responsabili il Consiglio editoriale della FUP e i Consigli scientifici delle singole collane. Le opere pubblicate nel catalogo FUP sono valutate e approvate dal Consiglio editoriale della casa editrice. Per una descrizione più analitica del processo di referaggio si rimanda ai documenti ufficiali pubblicati sul catalogo on-line (www.fupress.com).

\section{Consiglio editoriale Firenze University Press}

M. Garzaniti (Presidente), M. Boddi, A. Bucelli, R. Casalbuoni, A. Dolfi, R. Ferrise, M.C. Grisolia, P. Guarnieri, R. Lanfredini, P. Lo Nostro, G. Mari, A. Mariani, P.M. Mariano, S. Marinai, R. Minuti, P. Nanni, G. Nigro, A. Perulli.

$\boldsymbol{\partial}_{\text {L'edizione digitale on-line del volume è pubblicata ad accesso aperto su www. }}$ fupress.com.

La presente opera è rilasciata nei termini della licenza Creative Commons Attribution 4.0 International (CC BY 4.0: http://creativecommons.org/licenses/ by/4.0/legalcode). La licenza permette di condividere l'opera, nella sua interezza o in parte, con qualsiasi mezzo e formato, e di modificarla per qualsiasi fine, anche commerciale, a condizione che ne sia menzionata la paternità in modo adeguato, sia indicato se sono state effettuate modifiche e sia fornito un link alla licenza.

(C) 2019 Firenze University Press

Pubblicato da Firenze University Press

Firenze University Press

Università degli Studi di Firenze

via Cittadella, 7, 50144 Firenze, Italy

www.fupress.com

This book is printed on acid-free paper

Printed in Italy 


\section{Sommario}

9 Presentazione

Giusto Puccini

17 Senza lavoro? Cambiamento tecnologico e nuove sfide per la società

Franca Alacevich

57 Bibliografia

Franca Alacevich, Senza lavoro? Cambiamento tecnologico e nuove sfide per la società, (c) 2019 FUP, CC BY 4.0 International, published by Firenze University Press (www.fupress.com),

ISSN 2704-5935 (online), ISBN 978-88-5518-006-1 (online PDF) 



\section{$\underset{\substack{\text { Giusto } \\ \text { pucciNI }}}{\text { Presentazione }}$}

La Collana Lezioni e Letture della Facoltà di Scienze Politiche "Cesare Alfieri" dell'Università di Firenze, nata nel 2010, è proseguita e prosegue come collana dell'omonima Scuola.

La Scuola di Scienze Politiche "Cesare Alfieri”, del resto, è erede diretta della Facoltà, della sua peculiare e consolidata offerta formativa, nonché della sua tradizione quanto mai illustre nel panorama universitario nazionale ed internazionale.

Si tratta, appunto, della tradizione risalente addirittura a quella Scuola di Scienze Sociali, fondata a Firenze nel lontano 1875, che costituisce il più antico istituto italiano, ed uno dei più antichi in Europa, nel settore dell'insegnamento delle scienze politiche e sociali. Una lunga tradizione interdisciplinare, che ha visto collaborare fra loro figure assai auto- 
Giusto PUCCINI

revoli di storici, giuristi, politologi, sociologi ed economisti.

Orbene, le Lezioni, propriamente destinate all'inaugurazione dei vari anni accademici, e le Letture, costituiscono due appuntamenti annuali che intendono valorizzare proprio questa tradizione, coinvolgendo nella trattazione di tematiche di notevole interesse scientificoculturale e, insieme, di particolare attualità, secondo molteplici approcci disciplinari, docenti della Scuola (le Lezioni) e studiosi od esperti di grande valore italiani e stranieri (le Letture).

La Lezione inaugurale dell'anno accademico 2018/2019 è stata affidata a Franca Alacevich, professore ordinario di Sociologia del lavoro presso l'Università di Firenze, ed in particolare titolare, nell'ambito della Scuola "Cesare Alfieri”, degli insegnamenti di Sociologia del lavoro nel Corso di laurea in Scienze Sociali, e di Sociologia del lavoro in Europa nel Corso di laurea magistrale in Sociologia e ricerca sociale.

Le tematiche oggetto della sua vasta e qualificata produzione scientifica, così come delle numerose ricerche nazionali ed europee da lei dirette e/o coordinate, dimostrano indiscutibilmente che, con Franca Alacevich, ci troviamo 
di fronte ad una vera e propria autorità, a livello nazionale e non solo, in ambito sociologico e, in particolare, in materia di sociologia del lavoro.

Di Franca Alacevich, peraltro, non si può fare a meno di sottolineare anche i molteplici impegni di natura istituzionale assolti nel corso del tempo in ambito accademico con notevole generosità $\mathrm{e}$, insieme, con estrema competenza ed efficacia: fra i quali, in particolare, quello di delegato del Rettore dell'Università di Firenze per le Relazioni sindacali dal 1999 al 2006, quello di preside della Facoltà fiorentina di Scienze Politiche dal 2006 al 2012, quello di presidente della Conferenza dei Presidi delle Facoltà di Scienze Politiche dal 2009 al 2013, e quello di direttore del Dipartimento di Scienze Politiche e Sociali dell'Ateneo fiorentino dal 2009 al 2013.

Il tema da lei affrontato nella Lezione, d'altra parte, ovverosia Senza lavoro? Cambiamento tecnologico e nuove sfide per la società, risulta con ogni evidenza non solo di straordinaria attualità, ma anche dotato di una spiccata valenza multidisciplinare.

Si tratta, appunto, del tema relativo all'impatto occupazionale dei mutamenti tecnologici inerenti ai sistemi produttivi. 
Giusto PUCCINI

Orbene, dalla Lezione, emerge intanto come il tema in questione presenti anche una sua rilevante dimensione storica, essendo stato consapevolmente affrontato da autorevolissimi studiosi già a partire dalla seconda metà del XVIII secolo (Adam Smith) e dai primi decenni del XIX (David Ricardo). Successivamente, d'altronde, esso è stato autorevolmente ripreso e sviluppato prima, nel corso dell'Ottocento, da Karl Marx, e poi, nella prima metà del Novecento, da Frederick Taylor, da John Ford e da John Maynard Keynes. Dopodiché, esso ha costituito oggetto di rinnovata e aggiornata riflessione scientifica negli ultimi decenni del Novecento, «in corrispondenza con l'affacciarsi della terza rivoluzione industriale [...] contrassegnata dallo sviluppo delle ICT», ed infine nel primo scorcio di questo secolo, in coincidenza con l'avvio e con l'affermazione della quarta rivoluzione industriale, caratterizzata da «un'evoluzione molto avanzata delle tecnologie ICT».

D’altro canto, la Lezione di Franca Alacevich non ha soltanto il merito di evidenziare come, nel corso di questo periodo di oltre due secoli, sul tema in questione, sia ogni volta 
puntualmente emersa una contrapposizione fra 'pessimisti ed ottimisti': ovverosia fra studiosi tendenti a sottolineare le implicazioni negative dei mutamenti tecnologici sui livelli occupazionali e/o sulla qualità della stessa occupazione, e studiosi tendenti, al contrario, a sottolinearne le implicazioni positive.

Nella Lezione, infatti, si provvede anche, altrettanto meritoriamente, a dimostrare come generalmente, tanto dalle posizioni pessimistiche quanto da quelle ottimistiche, venga ad emergere, in misura più o meno accentuata, il carattere tutt'altro che meccanico e deterministico di dette implicazioni.

Di qui, dunque, una serie complessa di incognite e di variabili destinate a suggerire una conclusione alquanto problematica sul tema, tendente evocarne fino in fondo sia la dimensione economico-sociale che quella più squisitamente politico-istituzionale.

Oggi infatti, secondo Alacevich, «non si può sapere se la nuova rivoluzione tecnologica creerà più lavoro di quanto ne distruggerà, o viceversa [...] perché ogni stima si basa su dei presupposti che riguardano e prefigurano alcune delle possibili strategie degli attori 
Giusto PUCCINI

sociali (imprese, soggetti individuali e collettivi, politica)».

In specie, una fuoriuscita virtuosa e soddisfacente dalle trasformazioni del mercato del lavoro indotte da tale rivoluzione sembra decisamente dipendere dalla capacità da parte degli Stati di far fronte, mediante politiche adeguate anche dal punto di vista finanziario, ad una serie molteplice di esigenze fondamentali: da quella del potenziamento della «qualità della formazione di base e della formazione continua» a quella dello «sviluppo dei territori [...] più colpiti dai cambiamenti», da quella di un'incisiva "valorizzazione economica e commerciale delle innovazioni» a quella di un efficace sostegno economico e sociale delle categorie di soggetti più esposte alla perdita del lavoro e del relativo reddito.

E ciò vale tanto più per il nostro Paese, laddove simili ordini di esigenze, per l'appunto, sembrano manifestarsi, oggi non meno che in passato, in termini particolarmente accentuati. 


\section{Lettura}

$$
\text { "Cesare Alfieri" }
$$





\section{\begin{tabular}{r|} 
Franca \\
EvICH
\end{tabular} \\ Cambiamento \\ tecnologico e nuove sfide per la società}

Ogni innovazione tecnologica, dall'antichità ad oggi, produce effetti sul lavoro perché modifica l'insieme delle competenze necessarie per svolgere le diverse attività di produzione di beni e servizi, riduce il numero di alcuni tipi di occupazione mentre ne crea di nuovi. L'argomento proposto per questa Lezione ha dunque una lunga storia, ma qui ci si concentrerà soprattutto sugli effetti delle più recenti innovazioni tecnologiche, alimentate dall'intelligenza artificiale - cui spesso ci si riferisce con il termine di nuova, quarta, rivoluzione industriale.

La domanda messa a titolo è la domanda che molti si fanno in questo periodo, che ritorna costantemente sui mezzi di comunicazione, e nel dibattito pubblico, politico e scientifico. 
Franca ALACEVICH

Che cosa sta accadendo nel mondo del lavoro e sul fronte delle occupazioni? Il processo di spinta automazione, l'avanzato stadio della robotica, la possibilità di far camminare automobili e treni senza guidatori, di lavorare e comunicare in qualsiasi posto e con qualsiasi altra persona, comprimendo i vincoli di spazio e di tempo, stanno sostituendo molto lavoro umano con macchinari e processi intelligenti? Stanno riducendo drasticamente i posti di lavoro disponibili?

In queste brevi considerazioni non si arriva certo a dare una risposta esaustiva. Tuttavia, alcune riflessioni aiutano a evitare due semplificazioni, tra loro opposte ed entrambi largamente diffuse.

Da un lato, infatti, molti fanno previsioni pessimistiche, e a volte addirittura apocalittiche, sostenendo che la nuova, quarta, rivoluzione tecnologica sta già eliminando molte occasioni di lavoro, e ancor più ne distruggerà inesorabilmente nel prossimo futuro, e che dovremo abituarci a una società senza lavoro, o forse più appropriatamente - con sempre meno lavoro. Sul piano della qualità del lavoro, poi, queste previsioni si accompagnano alla consi18 
Senza lavoro?

Cambiamento

tecnologico e nuove sfide per

la società

derazione che il lavoro, il lavoro che resterà disponibile, subirà necessariamente un processo di dequalificazione: con procedimenti affidati a macchinari intelligenti, le competenze più elevate saranno riservate a un numero ristretto di ricercatori, programmatori, supervisori, con elevate competenze; mentre alla maggioranza dei lavoratori saranno richiesti compiti a bassa qualificazione, prevalentemente di servizio.

Altri sostengono, però, posizioni opposte, più ottimistiche e a volte persino entusiastiche. Considerando come nel passato ogni cambiamento tecnologico abbia creato nuove occupazioni in grado di rimpiazzare il lavoro che nel frattempo veniva distrutto, ritengono che così stia avvenendo anche oggi e che, anzi, le recenti innovazioni siano in grado di accrescere la qualità del lavoro per molti e di liberare $\mathrm{i}$ lavoratori del futuro dai compiti più gravosi $\mathrm{e}$ meno gratificanti.

Queste due posizioni si sono storicamente rivelate entrambi insoddisfacenti. Perché? Anzitutto perché quella dei pessimisti e degli apocalittici, almeno finora, è stata smentita dal fatto che se molti vecchi lavori si sono persi altri nuovi lavori li hanno sostituiti, e 
Franca ALACEVICH che alcuni vecchi lavori non sono spariti ma hanno cambiato fisionomia. Dall'altra parte, la visione genericamente ottimistica appare insoddisfacente perché rimanda a una sorta di meccanismo di riequilibrio automatico che appare poco credibile: per alcuni - per esempio, per numerosi esponenti della teoria economica - il riequilibrio avrebbe a che fare con il buon funzionamento del mercato, per altri invece dipenderebbe da una capacità di auto-aggiustamento, quasi deterministico, del sistema sociale. Entrambi questi processi sono, però, tutti da verificare nella realtà.

La tesi che si cercherà di argomentare in questa Lezione è che il futuro non è facilmente prevedibile, che ogni previsione è rischiosa perché il concreto sviluppo dei processi sociali dipende dall'agire dei soggetti e dei gruppi sociali, dalla consapevolezza e dalla capacità di intervento con le quali gli individui e le collettività cercano di rispondere alle sfide che di volta in volta il cambiamento tecnologico pone loro di fronte.

In realtà, le conseguenze del cambiamento tecnologico possono essere più o meno gravi o più o meno positive, nel tempo e nello spazio, 20 
Senza lavoro?

Cambiamento

tecnologico e nuove sfide per

la società

proprio in relazione a questa loro capacità, senza alcun automatismo.

Le visioni apocalittiche e ottimistiche hanno caratterizzato tutte le principali fasi di cambiamento tecnologico - dalla prima all'attuale, quarta, rivoluzione industriale - e da questo prenderà le mosse la Lezione. Si richiameranno, poi, alcune stime e alcune previsioni sui cambiamenti nel mercato del lavoro che sono state avanzate negli ultimi anni per immaginare il prossimo futuro. Infine, si proverà a trarre qualche indicazione, in termini di ipotesi, sul processo di cambiamento in atto, sugli effetti che può avere e su come si può cercare di guidarlo, e quindi sul ruolo delle politiche e degli attori sociali, sia individuali che collettivi.

\section{Visioni apocalittiche e visioni ottimistiche}

L'affacciarsi di due visioni contrapposte - pessimiste e apocalittiche o, al contrario, ottimistiche ed entusiastiche - non è affatto nuovo. E una questione antica. È una questione presente ancor prima dei grandi e veloci cambiamenti tecnologici che hanno accompagnato la 
Franca ALACEVICH

rivoluzione industriale nelle sue diverse fasi. E una questione ancor più presente, tuttavia, da quando le nostre società si sono avventurate sulla strada dell'industrializzazione e soprattutto da quando gli sviluppi delle scienze applicate, negli ultimi decenni, sono divenuti sempre più rapidi e pervasivi.

Si può rifarsi, a titolo di esempio e brevemente, alle visioni contrapposte che già si manifestavano tra fine Settecento e primo Ottocento, agli albori della prima rivoluzione industriale, quando le scoperte consentono di avviare la meccanizzazione del settore tessile, in particolare l'industria del cotone, e, a breve distanza, la macchina a vapore cambia radicalmente il modo di produzione nei settori metallurgico, dei trasporti, e in generale in tutta l'industria manifatturiera.

Già in questa fase, infatti, si iniziano a rintracciare le due visioni opposte di coloro che vedono in questo cambiamento una iattura, perché le nuove macchine eliminano lavoro umano, e rendono sempre meno qualificato il lavoro che resta disponibile, e di coloro che, al contrario, ritengono che nuove e migliori occasioni di lavoro si aprono a causa dell'in- 
Senza lavoro?

Cambiamento

tecnologico e nuove sfide per

la società

troduzione delle innovazioni nei sistemi produttivi.

Per fare solo qualche esempio, alla fine del Settecento Adam Smith, nel suo La ricchezza delle nazioni (1776), ascrivendosi tra gli ottimisti, ritiene che i nuovi sistemi produttivi rispondano all'esigenza di semplificare e velocizzare il lavoro e, infatti, le principali innovazioni traggono origine proprio dagli stessi operai. Con le sue parole:

gran parte delle macchine che sono usate in quelle industrie in cui il lavoro è più suddiviso in origine furono invenzioni di operai comuni $\mathrm{i}$ quali, essendo addetti a qualche semplicissima operazione, rivolsero la loro attenzione a trovare metodi più facili e più rapidi per eseguirla (p. 263).

Ancor prima, nelle Lezioni di Glasgow (1763), Smith sostiene che il motivo che spinge il singolo lavoratore a introdurre miglioramenti è quello di alleviare la fatica:

tutta l'attenzione sarà rivolta a come realizzare [le cose] nel miglior modo [...] Probabilmente fu un contadino a inventare l'aratro [...] qualche misero schiavo, occupato per lungo tempo 
Franca ALACEVICH

a macinare il grano tra due sassi, sarà stato il primo a trovare il modo di fissare il sasso superiore ad un perno (p. 230).

Dunque, il cambiamento è stimolato dalla ricerca di migliorare la qualità del lavoro da parte degli stessi lavoratori.

Smith non si ferma a questa considerazione sulla qualità del lavoro. Ritiene anche che l'introduzione della meccanizzazione possa creare tanto lavoro quanto ne risparmia. La logica del sistema capitalistico, infatti, fa sì che: «un maggior numero di cervelli si dedica all'invenzione delle macchine più adatte per eseguire il lavoro di ciascuno, e crescono così le possibilità che ne siano inventate molte» (1776, p. 312).

In effetti, l'impressionante crescita del numero di brevetti in Gran Bretagna ne è testimone. La storica inglese Phyllis Mary Deane, nel suo La prima rivoluzione industriale (1965), ne riporta i valori: dai 56 brevetti registrati nel periodo 1730-1739 si arriva, in un secolo soltanto, ai 4581 del periodo 1840 1849. Questa enorme crescita è costante nel periodo, ma assume un'accelerazione vorticosa nel giro di boa del secolo, tra Settecento e Ottocento (più in particolare, tra il 1780 e il 
Senza lavoro?

Cambiamento

tecnologico e nuove sfide per

la società

1810), quando il numero dei brevetti in Gran Bretagna quasi triplica ${ }^{1}$.

Mentre cresce la prosperità, aumenta l'interdipendenza tra i soggetti dediti a diversi compiti, aumenta anche la richiesta di beni e servizi perché cresce la densità della popolazione e il lavoro si viene specializzando, si allargano gli scambi commerciali per il miglioramento dei trasporti, e dunque aumenta la quantità di occupazioni necessarie per soddisfare tutte queste esigenze. In sintesi, secondo Smith, se il mercato interno e internazionale è in grado di assorbire tutte le merci prodotte in crescente quantità, per l'aumento della produttività, non si manifesta una disoccupazione tecnologica.

Smith è un esempio illustre di come il cambiamento tecnologico viene visto essenzialmente in una chiave positiva e non

${ }^{1} \mathrm{Nel}$ «decennio 1760-70 [...] per la prima volta il numero dei brevetti rilasciati [in Inghilterra] superò la cifra di 200 [...] Da allora in poi, esso aumentò costantemente, ad un tasso di circa il 50 per cento per decennio [...] [a metà ' 800 ] rispetto al 1760 il numero dei brevetti risultava 20 volte maggiore» (p. 172). 
Franca ALACEVICH

problematica per la società, a condizione che l'economia di mercato funzioni efficacemente.

Tuttavia, nello stesso periodo, molti la pensano diversamente. L'introduzione di apparecchi moderni nell'industria, capaci di semplificare e velocizzare il lavoro operaio e di ridurne la complessità, fino al limite di richiedere quasi esclusivamente forza lavoro non qualificata, trova resistenze negli operai. L'esempio più noto è quello del movimento luddista, che trae il nome da Ned Ludd, un operaio tessile che rifiutava l'introduzione della spinnig-jenny ${ }^{2} \mathrm{e}$ del telaio meccanico, perché sottraevano lavoro ai lavoranti a domicilio portandolo nelle nuove fabbriche, con forte risparmio di forza lavoro. Le prime rivolte sono del 1811-1812 e vedono il sabotaggio dei telai meccanici e dei

${ }^{2}$ La spinning-jenny, in italiano 'giannetta', era una macchina filatrice a lavoro intermittente e dotata di fusi multipli, inventata attorno al 1765 a Stanhill in Inghilterra da James Hargreaves. Questa macchina consentiva di sostituire il lavoro di più operai, inizialmente otto, con un solo lavoratore che poteva controllare contemporaneamente più processi. 
Senza lavoro?

Cambiamento

tecnologico e nuove sfide per

la società

nuovi macchinari industriali. Suscitano ampia discussione, da un lato per gli atti violenti messi in essere dai rivoltosi e per la dura reazione del potere costituito, dall'altro lato per la messa in evidenza della drammatica situazione di sfruttamento e delle pessime condizioni di vita dei lavoratori. La questione arriva anche alla $\mathrm{Ca}$ mera dei Lords, con gli interventi appassionati di Lord Byron in favore degli operai.

Tuttavia, non sono solo gli operai o i politici a paventare gli effetti negativi della rivoluzione industriale. È interessante notare che un altro tra i padri fondatori dell'economia, David Ricardo, nei suoi Principi di economia politica e della tassazione (1817), prevede la possibilità di una disoccupazione da meccanizzazione:

Sono convinto che la sostituzione delle macchine al lavoro umano sia spesso assai dannosa agli interessi della classe dei lavoratori [...] Una parte dei suoi membri verrà allontanata dal lavoro [...] L'opinione nutrita dalla classe lavoratrice, secondo cui l'impiego delle macchine è spesso dannoso ai suoi interessi, non è fondata sul pregiudizio e sull'errore, ma è conforme ai corretti principi dell'economia politica (pp. 295, 297, 299). 
Franca ALACEVICH

Per Ricardo, quindi, il processo di aggiustamento rispetto alla crescente meccanizzazione dei sistemi produttivi sembra più problematico rispetto a come lo vedeva Smith. Ricardo, semplificando molto, sostiene che l'aumento della produttività può favorire l'accumulazione di capitale a costi minori, riducendo soprattutto il costo del lavoro, e dunque, se non si verifica una crescente domanda di beni e servizi tale da compensare l'accresciuta produttività, si possono verificare effetti negativi sul volume della forza lavoro.

Dunque, in questa prima fase del cambiamento tecnologico il confronto tra pessimisti e ottimisti si manifesta già con chiarezza. Tuttavia, su un punto entrambe le posizioni convergono. Gli effetti positivi o negativi del cambiamento tecnologico non dipendono soltanto, meccanicamente o deterministicamente, dalle innovazioni e dal cambiamento tecnologico stesso ma sono legati anche, e soprattutto, ai comportamenti e alle strategie che gli attori sociali mettono in campo.

La seconda ondata di cambiamento arriva a fine Ottocento con l'introduzione dell'elettricità, dei motori elettrici che consentono ai 28 
Senza lavoro?

Cambiamento

tecnologico e nuove sfide per

la società

lavoratori più rapidità e più flessibilità nello svolgimento dei compiti, oltre che una crescita della scala della produzione e giornate lavorative che possono estendersi anche alle ore di buio. Contemporaneamente, l'elettricità modifica anche la vita domestica e sociale aprendo la strada a nuovi prodotti e servizi che pian piano si diffondono e richiedono di aumentarne la produzione. Nelle case entrano molti nuovi apparecchi: dal bollitore al ferro da stiro, dal forno alle stufe, alle aspirapolveri, ai ventilatori. Nella vita sociale, i servizi per il tempo libero e l'intrattenimento, così come i mezzi di informazione e comunicazione, non soltanto cambiano le abitudini della popolazione ma a loro volta impongono nuove produzioni di beni e di servizi. Progressivamente, poi, con innovazioni sul fronte dei prodotti chimici e l'uso del petrolio si apre la strada a ulteriori nuovi prodotti e servizi, fino ad arrivare alle automobili a larga diffusione.

In questa fase, di nuovo, le valutazioni degli studiosi e degli operatori si dividono tra coloro che vedono un pericolo per l'occupazione e la qualità del lavoro e coloro che, al contrario, sottolineano gli aspetti positivi su entrambi 
Franca

ALACEVICH

i fronti. Anche in questo caso, bastano due esempi.

Tra i pessimisti possiamo ascrivere Karl Marx, preoccupato dagli effetti della concentrazione del lavoro nelle aziende industriali meccanizzate sulla qualità del lavoro e sull'occupazione. L'aumento della produttività, unito alla dequalificazione degli operai divenuti mere appendici delle macchine e pertanto facilmente sostituibili, genera disoccupazione, e si viene a creare un esercito di disoccupati, l'esercito industriale di riserva, utile al sistema capitalistico. Le modalità di esercizio del lavoro rendono i lavoratori estranei al processo produttivo e ai risultati del proprio impegno, alienati economicamente e psicologicamente.

Marx, certamente un critico del sistema di produzione capitalistico, nel primo libro de $I l$ Capitale (1867) afferma:

le macchine, considerate in sé, abbreviano il tempo di lavoro mentre, adoperate capitalisticamente, prolungano la giornata lavorativa [...] in sé alleviano il lavoro e adoperate capitalisticamente ne aumentano l'intensità [...] in sé sono una vittoria sulla forza della natura e adoperate capitalisticamente soggiogano 
Senza lavoro?

Cambiamento

tecnologico e nuove sfide per

la società

l'uomo mediante la forza della natura [...] in sé aumentano la ricchezza del produttore e usate capitalisticamente lo pauperizzano (p. 486).

Non è, dunque, il progresso tecnologico in sé la fonte dei problemi ma l'uso che se ne fa da parte di coloro che detengono il potere economico.

Per contro, nei primi anni del Novecento si viene affermando un'altra visione, in cui il progresso tecnologico assume una connotazione positiva, sia per quanto riguarda la forza lavoro occupata sia per quanto riguarda la qualità del lavoro.

Uno studioso che sottolinea gli aspetti positivi del cambiamento tecnologico, soprattutto sul fronte della qualità del lavoro e della riduzione della fatica, come Frederick Taylor, li collega a un radicale cambiamento di atteggiamento dei datori di lavoro (L'organizzazione scientifica del lavoro, 1911). Per alleviare la fatica e aumentare la produttività, ogni operazione può e deve essere studiata a fondo, trovando il modo migliore di agire (the one best way), e dedicando tempo ed energie alla formazione di ciascun operatore. Tuttavia, la teoria di Taylor resta inapplicata. Henry Ford, che applica a suo mo- 
Franca ALACEVICH

do i principi di Taylor, non si preoccupa però di cambiare il modo di agire di datori di lavoro e lavoratori quanto di organizzare il lavoro per aumentare i profitti, portando il lavoro all'operaio e non l'operaio al lavoro, con la catena di montaggio (La mia vita e la mia opera, 1922).

Per Taylor e Ford, comunque, le nuove tecnologie sono viste come un vero e proprio progresso, senza effetti negativi sull'occupazione, perché l'aumento della produttività avrebbe aumentato la massa dei beni prodotti; beni peraltro standardizzati e a basso costo, quindi accessibili a sempre più larghi strati di popolazione e quindi in grado di essere smerciati e di richiedere sempre più lavoro per produrli. Entrambi non si preoccupano troppo della qualità del lavoro, della dequalificazione dei lavoratori che il sistema produttivo produce, vedendo soltanto gli effetti benefici legati alla riduzione della fatica.

Di nuovo, di fronte alla seconda rivoluzione industriale, troviamo dunque pessimisti e ottimisti. Tra questi però si affaccia una terza e diversa posizione. Di fronte alla Grande depressione del 1929 - in cui si sommano crisi economica, disoccupazione diffusa e intro- 
Senza lavoro?

Cambiamento

tecnologico e nuove sfide per

la società

duzione sempre più spinta di tecnologie che risparmiano mano d'opera - cresce la preoccupazione di politici e studiosi.

Tra questi ultimi, non si può non ricordare John Maynard Keynes. Keynes parte dalla considerazione della diversa velocità dei processi sociali: la velocità dei cambiamenti tecnologici e delle innovazioni è molto più rapida di quella del sistema sociale e delle istituzioni, molto più rapida della loro capacità di creare nuova occupazione e di riconvertire i lavoratori in modo che possano occupare le nuove posizioni di lavoro disponibili.

Keynes esprime meno fiducia dei suoi predecessori sulle capacità del mercato di promuovere l'adattamento dell'occupazione e sottolinea il ruolo cruciale di un attore come lo Stato, che può far crescere la domanda di beni e di servizi attraverso il ricorso alla spesa pubblica. La crescita della domanda - nel suo lessico la 'domanda aggregata' - fa aumentare l'occupazione:

una socializzazione di una certa ampiezza dell'investimento si dimostrerà l'unico mezzo per farci avvicinare alla piena occupazione [...] I controlli centrali necessari ad assicurare la piena occupazione richiederanno natural- 
Franca ALACEVICH

mente una vasta estensione delle funzioni tradizionali di governo [...] unico mezzo attuabile per evitare la distruzione completa delle forme economiche esistenti [...] condizione di un funzionamento soddisfacente dell'iniziativa individuale (1936, pp. 549, 550, 551).

Dunque, in questo caso l'attenzione si sposta sulle capacità di intervento attivo delle istituzioni pubbliche per affrontare i problemi dell'occupazione di fronte ai cambiamenti, soprattutto tecnologici, dell'organizzazione produttiva.

Anche in questa fase, come si vede, nessun risultato è meccanicamente determinabile e gli effetti del cambiamento tecnologico sul volume e la qualità del lavoro dipendono dagli attori sociali. Per Marx, il cambiamento tecnologico di per sé non è negativo, sono gli attori sociali a determinare la riduzione dell'occupazione e della qualità de lavoro, inserendolo in un sistema capitalistico. Per Taylor, gli effetti del cambiamento tecnologico possono essere positivi se e in quanto vi sia un accurato studio delle mansioni e un'accurata formazione dei lavoratori. Per Ford, meno interessato alla qualità del lavoro, gli effetti positivi si traducono in 
Senza lavoro?

Cambiamento

tecnologico e nuove sfide per

la società

maggiore possibilità di sempre più larghi strati di popolazione di acquisire beni di consumo personale e familiare resi accessibili da una produzione su larga scala e che ne riduce i costi. Per Keynes, infine, anche in tempi di difficoltà economica, il cambiamento tecnologico può essere messo a frutto da un intervento dello Stato, volto ad attutire gli effetti negativi dell'innovazione e della crisi e a giocare un ruolo proattivo, seppur temporaneo, nella società e nel mondo del lavoro.

Analoghe contrapposizioni tra pessimisti e ottimisti si verificano all'affacciarsi della terza rivoluzione industriale - contrassegnata dallo sviluppo delle ICT, Information and Communication Technologies, negli ultimi decenni del Novecento. Le valutazioni degli studiosi e degli operatori si dividono ancora una volta tra coloro che vedono un pericolo per l'occupazione e la qualità del lavoro e coloro che, al contrario, sottolineano gli aspetti positivi su entrambi i fronti.

Daniel Bell, nel suo The Coming of the Post-Industrial Society. A Venture in Social Forecasting (1973), enfatizza la crescita della domanda di servizi qualificati, di nuovi beni di 
Franca ALACEVICH

consumo, che promuovono una redistribuzione degli impieghi favorevole ai tecnici e ai professionisti, le cui conoscenze teoriche sono elevate e fonte di costante innovazione (parla di una nuova tecnologia intellettuale), con effetti sulla qualità del lavoro e saldi non necessariamente negativi dell'occupazione.

Jeremy Rifkin, alcuni anni dopo, intitola un suo libro La fine del lavoro (1995), imputandone la causa alla diffusione delle tecnologie informatiche, che riducono i posti di lavoro e aumentano le disuguaglianze tra i poco numerosi lavoratori più qualificati e i molti che svolgono lavori a bassa qualificazione:

le tecnologie intelligenti aumentano la produttività e si vengono sempre più a sostituire ai lavoratori [...] Come per il comparto manifatturiero, ci si aspetta che le tecnologie intelligenti riducano la forza lavoro dei colletti bianchi e dei servizi a una frazione delle sue attuali dimensioni (pp. XXII-XXIII).

Ma ancora una volta, come sempre, non vi è nulla di ineluttabile. L'esito dell'introduzione di innovazioni tecnologiche sulloccupazione e sulla qualità del lavoro dipende dall'azione dei soggetti sociali: imprese, lavoratori, consumatori, stato. 36 
Senza lavoro?

Cambiamento

tecnologico e nuove sfide per

la società

Gli effetti del nuovo cambiamento

tecnologico: ipotesi e stime

La nuova fase del cambiamento - che va sotto l'etichetta di quarta rivoluzione industriale è l'evoluzione molto avanzata delle tecnologie ICT. La caratterizzano, anzitutto, la digitalizzazione e l'Internet of things, ovvero la presenza di microprocessori all'interno degli oggetti, che mappano il mondo fisico e permettono di ridurre tempi e azioni umane: dal frigorifero che compra automaticamente il latte quando è finito alla sveglia che suona prima quando c'è traffico. Altre caratteristiche sono la disponibilità di big data e open data (un'enorme mole di dati eterogenei, strutturati e non, estrapolabili, analizzabili, che possono aiutare a scoprire i legami tra fenomeni diversi e prevederne di futuri); l'intelligenza artificiale (un sistema tecnologico che risolve problemi o svolge compiti e attività che rientrano tipicamente nelle abilità umane); il cloud computing (una tecnologia che permette di elaborare e archiviare dati in rete, consente l'accesso ad applicazioni e dati memorizzati su un hardware remoto, abbattendo i costi); le smart factories, ambienti pro- 
Franca ALACEVICH

duttivi in cui uomini e macchine cooperano avvalendosi di sistemi informatici in grado di interagire tra loro; lo smart working, ovvero la possibilità di controllare da remoto la produzione attraverso webcam e sensori disposti nei luoghi della produzione, o di lavorare in remoto nei settori non manifatturieri; e soprattutto lo sviluppo sempre più accelerato della robotica e le sue molteplici applicazioni.

Anche oggi, di fronte a questa nuova fase dell'innovazione tecnologica, possiamo trovare tesi contrapposte, che prefigurano scenari pessimistici e ottimistici. Ciascuna di queste tesi si basa su stime degli effetti occupazionali di questi cambiamenti; tutte le stime sono costruite con metodologie molto sofisticate e sono basate su solidi apparati teorici. Ciascuna mette in evidenza gli effetti qualità del lavoro a partire da ricerche empiriche sul mondo del lavoro. Tuttavia sono tesi che giungono a esiti contrapposti. A chi si deve credere? Quali sono più affidabili delle altre? Prima di provare a dare una risposta, è opportuno ricordarne qualcuna, a mo' di esempio.

Due noti studiosi inglesi di Oxford, Carl Benedikt Frey e Michael Osborne, nel saggio 38 
Senza lavoro?

Cambiamento

tecnologico e nuove sfide per

la società

The Future of Employment. How Susceptible are Jobs to Computerization (2017), analizzano la probabilità di 702 occupazioni codificate dal Department of Labor di essere computerizzate e svolte da macchine, con l'obiettivo di stimare i posti di lavoro a rischio. I loro risultati prevedono che l'introduzione negli Stati Uniti dei processi di automazione e digitalizzazione, dal machine learning alla robotica, elimineranno nel prossimo futuro quasi la metà degli attuali occupati negli USA:

Distinguiamo tra occupazioni ad alto, medio o basso rischio, in relazione alla probabilità che hanno di venire sostituite da computer [...] Secondo le nostre stime, circa il $47 \%$ dell'occupazione totale negli Stati Uniti si colloca nella categoria ad alto rischio. Ci riferiamo a queste come a posti di lavoro a rischio - ovvero, lavori che ci aspettiamo possano venire automatizzati presto, probabilmente nell'arco di dieci o venti anni (p. 268, trad. mia).

Catalogando le attività a seconda della diversa probabilità (bassa, media o alta) di venire sostituite, forniscono un elenco interessante di lavori ad alto rischio di venire sostituiti da computer. L'elenco comprende, per esempio, i 
Franca ALACEVICH

settori dei trasporti e della logistica, il lavoro amministrativo di ufficio, le professioni para legali, il mondo della produzione di beni. Più sorprendentemente, sono considerate ad alto rischio anche alcune professioni nella sanità, nei servizi, nelle costruzioni, dove già molte attività sono state computerizzate:

In primo luogo, il mercato dei robot per uso personale e domestico è già cresciuto di circa il 20\% l'anno. Mentre il vantaggio comparativo del lavoro umano in attività che richiedono mobilità e destrezza diminuirà nel tempo, il ritmo della sostituzione di lavoro nei servizi probabilmente aumenterà anche di più. In secondo luogo, anche se pare contro intuitivo che l'occupazione nel commercio, che richiede un elevato livello di intelligenza umana, sia soggetta a un'ondata di computerizzazione nel prossimo futuro, i lavori ad alto rischio nel settore includono, per esempio, quelli dei cassieri, degli addetti alla reception, degli impiegati nei noleggi, degli addetti al telemercato. Anche se questi lavori richiedono compiti di relazione, non implicano necessariamente alti livelli di intelligenza umana [...] In terzo luogo, la tecnica di pre-fabbricazione consentirà a una quota crescente di lavoro nelle costruzioni di 
Senza lavoro?

Cambiamento

tecnologico e nuove sfide per

la società

essere svolta in fabbrica, eliminando parte della variabilità dei compiti. Questo trend può portare alla computerizzazione del lavoro nel settore (p. 265, trad. mia).

Applicando lo stesso metodo di analisi, altri ricercatori tedeschi hanno stimato leffetto in Germania molto vicino al dato statunitense (42\%).

Questo lavoro, molto discusso, si accompagna ad altri che lo sostengono. Il World Economic Forum (in The Future of Jobs. Employment, Skills and Workforce Srategy for the Fourth Industrial Revolution, 2016) prevede che già nel vicinissimo 2020 saranno perduti più di sette milioni di posti di lavoro, a fronte di due milioni creati, con un preoccupante saldo negativo. Per di più, gli effetti di questo processo rischiano di essere molto pesanti sul già fragile lavoro femminile, perché quasi la metà dei posti perduti (48\%) riguarderebbe le occupazioni poco qualificate nei servizi, generalmente a predominanza femminile: i posti persi per gli uomini sarebbero quattro milioni a fronte di un milione e quattrocentomila nuovi posti; per le donne i posti persi sarebbero tre milioni a fronte di mezzo milione circa di nuovi posti (Tonarelli, 2018). 
Franca ALACEVICH

Altri due studiosi del Massachusetts Institute of Technology di Cambridge, Mass., Erik Brynjolfsson and Andrew McAfee, nel loro The Second Machine Age (2014), arrivano qualche anno prima ad analoghe considerazioni. Pur convinti che l'entrata «in una seconda età delle macchine» porterà trasformazioni "profondamente benefiche» (p. 17), su un punto ritengono che si creeranno problemi, il lavoro. Con le loro parole:

La digitalizzazione rapida e in via di accelerazione porterà probabilmente devastazioni economiche più che ambientali, dovute al fatto che, diventando i computer più potenti, le aziende avranno meno bisogno di certi tipi di dipendenti. Nella sua corsa il progresso tecnologico lascerà a piedi qualcuno, forse tanta gente. Come dimostreremo, non c'è mai stato un momento migliore per essere un lavoratore specializzato o istruito nel senso giusto del termine, perché questo è il tipo di persona che può usare la tecnologia per creare e catturare valore. Però, non c'è mai stato un momento peggiore per essere un lavoratore che ha da offrire soltanto capacità 'ordinarie' perché computer, robot, e altre tecnologie digitali stanno acquisendo le medesime capacità e competenze a una velocità inimmaginabile (p. 18). 
Senza lavoro?

Cambiamento

tecnologico e nuove sfide per la società

Per contro, un altro economista dello stesso istituto, il Massachusetts Institute of Technology di Cambridge Mass., David Autor, contrasta questa automation anxiety diffusa. $\mathrm{Ha}$ più volte scritto negli ultimi anni che l'innovazione distrugge posti di lavoro ma ne crea al contempo altri, in altri settori, e così è sempre stato. Il titolo di un suo brillante saggio è chiaro: Why Are Still So Many Jobs? The History and Future of Workplace Automation (2015). Le nuove tecnologie hanno, infatti, bisogno di lavoro umano, che è complementare al loro sviluppo:

Lautomazione infatti sostituisce il lavoro - è questo tipicamente il suo obiettivo. Tuttavia, l'automazione è anche complementare al lavoro, aumenta la produzione in modi che portano a una maggiore domanda di manodopera [...] e interagisce con gli adeguamenti della forza lavoro. Infatti, punto centrale del paper è che i giornalisti e persino i commentatori più esperti tendono a sovrastimare la sostituzione di lavoro umano da parte delle macchine e non tengono conto della forte complementarietà tra automazione e lavoro che aumenta la produttività, i guadagni, e la domanda di lavoro stessa (p. 5, trad. mia). 
Franca ALACEVICH

Per esempio, nella grande distribuzione le casse automatiche levano lavoro ai cassieri e le ordinazioni online ai commessi ma creano nuovi lavori: di più elevata qualità, per i tecnici che disegnano e manutengono i nuovi macchinari e i siti web; e di più bassa qualità, come si vede nella distribuzione in cui appaiono i cosiddetti riders di cui in questi giorni si parla molto.

Inoltre, le nuove tecnologie hanno dei limiti intrinseci, che riguardano tutte le attività che Autor definisce 'non-routine'. I compiti routinari seguono ben note e precise procedure che possono essere codificate dai software, digitalizzate ed effettuate da macchinari. Le attività non-routine, al contrario, hanno bisogno dell'intelligenza umana, perché richiedono flessibilità, capacità di valutazione, capacità senso-motorie, intuizione, creatività, capacità espressive, buon senso - tutte competenze che si acquisiscono tacitamente. Richiamando il paradosso di Michael Polanyi (The Tacit Dimension,1966) sulla conoscenza tacita - "we know more than we can tell" - Autor sottolinea, dunque, come alcune attività non possano essere trasmesse ai computer. Il suo esempio 
Senza lavoro?

Cambiamento

tecnologico e nuove sfide per

la società

della sedia è suggestivo (p. 25). Perché una macchina possa riconoscere una sedia è necessario fornirle le caratteristiche: gambe, braccioli, sedile, schienale. Ma molte sedie non hanno tutti questi elementi, tuttavia se ne eliminiamo alcuni il computer identificherà come sedie anche molti altri oggetti, per esempio dei tavolini. Quel che un bambino può immediatamente riconoscere diviene un compito impossibile per una macchina.

Autor conclude ottimisticamente, ritenendo che il processo di sostituzione di lavoro umano con macchinari non possa continuare indefinitamente:

La mia previsione è che questa polarizzazione del lavoro non potrà continuare indefinitamente [...] Mi aspetto che un rilevante strato di lavori che richiedono competenze di medio livello, e che uniscono competenze tecnicoprofessionali con livelli medi di alfabetizzazione, capacità di calcolo, adattabilità, problem solving e buon senso, resteranno in vita per decenni. La mia ipotesi è che molti dei compiti incorporati in questi lavori non possono essere esternalizzati - con macchinari che svolgono compiti di medio livello e lavoratori impiegati 
Franca ALACEVICH

soltanto nelle attività residuali a basse competenze - senza una sostanziale caduta della qualità (pp. 26-27, trad. mia).

Nei lavori non-routine, infatti, i lavoratori hanno grandi vantaggi comparativi: capacità di interazione interpersonale e problem solving, flessibilità, adattabilità. In molte attività, per di più, la relazione face-to-face resta indispensabile.

Va poi considerato che lo sviluppo delle moderne tecnologie consente di ottenere alta qualità della produzione anche senza seguire la via bassa del risparmio sui costi, che porta alla delocalizzazione delle attività. Riportare in casa molte lavorazioni prima delocalizzate all'estero può contribuire ad aumentare l'occupazione domestica.

Ampiamente citato è il caso del ritorno in Germania, ad Ansbach, di larga parte della produzione di Adidas, da più di vent'anni delocalizzata in Cina e in Vietnam, con la contestuale chiusura di nove dei dieci stabilimenti tedeschi. Dando vita a una nuova fabbrica - la Speedfactory - altamente automatizzata, con robot che preparano e cuciono le scarpe da ginnastica, si sono creati nuovi posti di lavoro 46 
Senza lavoro?

Cambiamento

tecnologico e nuove sfide per

la società

in Germania e si è avvicinata la produzione alle sedi di vendita.

Da queste considerazioni, si vede come le due rappresentazioni, pessimistiche e ottimistiche, si confrontino anche oggi. Torna dunque il quesito: come valutare la situazione in cui ci troviamo? Il cambiamento tecnologico dei prossimi anni distruggerà lavoro? o sarà anche in grado di creare nuovo lavoro? E soprattutto, quale sarà il saldo generale?

Oggi è forse impossibile prevederlo. Molto dipende dalle strategie delle imprese, degli attori sociali, e della politica, e persino dei consumatori.

\section{Riflessioni conclusive. Chi sarà} avvantaggiato e chi svantaggiato? È possibile governare questo processo?

I numeri, come si è visto, sono incerti, ma anche se fosse più chiaro quel che avverrà sul volume dell'occupazione resterebbero molti problemi aperti. I numeri, infatti, non bastano. Che il saldo occupazionale sia positivo o negativo non dice nulla sulle persone che perderanno lavoro 
Franca ALACEVICH

né su quelle che lo potranno trovare; non dice nulla sulle possibilità per chi perde il lavoro di riconvertirsi e trovare una nuova occupazione.

Quando si verificano grandi cambiamenti nel mondo del lavoro, ci sono sempre gruppi di persone che si trovano più in difficoltà e altri gruppi di persone che si trovano in posizione più favorevole. E questi due gruppi non coincidono, solitamente, e possono non essere negli stessi luoghi.

Come si è detto, gli aggiustamenti rispetto alle sfide poste dall'innovazione tecnologica non avvengono automaticamente. Dunque, non si può dare per scontato che chi perde un'occupazione in seguito a questi cambiamenti possa affrontare, individualmente, i rischi che ne discendono. Si richiedono interventi pubblici a sostegno dell'azione individuale, per limitare i costi per i singoli soggetti ma anche per la società nel suo complesso, che vede svalutato il suo capitale umano, e quindi le sue possibilità di sviluppo e di coesione sociale, e che rischia di dover sopportare costi aggiuntivi per far fronte ai problemi sociali che ne discendono (dalle spese sanitarie a quelle assistenziali, per far solo due esempi).

48 
Senza lavoro?

Cambiamento

tecnologico e nuove sfide per

la società

Da qui, evidentemente, l'importanza delle politiche per la formazione e la riqualificazione professionale, così come delle politiche per lo sviluppo dei territori che sono più colpiti dai cambiamenti. Infatti, in una visione più tradizionale dei processi di aggiustamento, basata sul mercato, si può ipotizzare che il più basso costo del lavoro di regioni o paesi arretrati attiri gli investimenti verso queste aree. In realtà, si vede come l'innovazione tecnologica spinga in direzione di una maggiore concentrazione degli investimenti e dell'occupazione nelle aree più sviluppate, dove è migliore il capitale umano e sono più estese le possibilità di rapporti tra mondo della ricerca e delle imprese, e più in generale le infrastrutture materiali e immateriali. L'investimento volto ad accrescere le conoscenze e le competenze, da un lato, e il livello di produttività unito alla propensione verso l'innovazione, dall'altro lato, stanno tra loro in uno stretto rapporto: si possono rafforzare vicendevolmente in un circolo virtuoso o, al contrario, possono contribuire a ridurre le possibilità di crescita dell'economia, e dunque del mercato del lavoro, in un circolo che diviene vizioso. 
Franca ALACEVICH

Anche in questo caso, quindi, i processi di aggiustamento tra i territori non avvengono automaticamente ma richiedono interventi specifici, in mancanza dei quali le disuguaglianze territoriali tendono ad accentuarsi.

Guardando all'Italia, in questa prospettiva, diviene allora cruciale porre attenzione in termini di spesa e alla qualità della formazione di base e della formazione continua. Per quanto riguarda l'istruzione, sono note le carenze del nostro paese. Un recente rapporto dell'OECD (Education at a Glance, 2018) indica come su cento italiani adulti (dai 25 ai 64 anni) solo diciotto sono laureati, triennali o magistrali. Nella media dei 35 paesi OECD sono invece il doppio (36\%). Solo il Messico ne ha meno dell'Italia. Inoltre, solo un quarto dei laureati ha fatto studi scientifici, un dato inferiore alla media OECD ed europea, contro il 35\% della Germania, per esempio. Anche per la formazione continua e la riqualificazione degli adulti il paese è agli ultimi posti tra i Paesi sviluppati per livello di spesa. Naturalmente, resta da considerare poi il problema della qualità della formazione.

Questi dati preoccupano molto, perché mostrano come non ci si stia attrezzando per 
Senza lavoro?

Cambiamento

tecnologico e nuove sfide per

la società

giocare un ruolo da protagonisti in questo processo e non si stiano preparando soggetti capaci di inserirsi nei nuovi posti di lavoro che si stanno creando.

Come si diceva, occorre anche porre attenzione a dove il lavoro - generalmente poco qualificato - si perde, e a dove il lavoro - altamente qualificato - si crea. Le nuove tecnologie richiedono molto lavoro di ricerca e sviluppo, di produzione e sviluppo di nuovi macchinari e di sistemi informativi molto sofisticati.

L'Italia è un paese in cui non è mai mancato l'ingegno creativo e la capacità di generare innovazione, purtroppo però spesso non si riesce a sostenere la valorizzazione economica e commerciale delle innovazioni. Tre esempi del passato aiutano a ricordarlo.

Il telefono fu inventato da Antonio Meucci che nel 1854 realizzò il primo esemplare in casa sua, o da Alexander Bell, che lo brevettò nel 1876 ? Meucci non aveva i soldi necessari a brevettare la sua scoperta in Italia e i soldi per mantenere il suo brevetto provvisorio, del 1871, in America. La querelle è durata più di un secolo. Solo nel 2002 il Congresso degli Stati Uniti d'America ha riconosciuto la paternità di 
Franca ALACEVICH

Meucci. Durante questo secolo, lo sviluppo di quell'innovazione e la sua industrializzazione sono stati portati avanti in paesi diversi dall'Italia, con enormi vantaggi.

La lampadina fu inventata da Alessandro Cruto, un inventore piemontese che di mestiere faceva il capo mastro, o dall'inglese Joseph Swan, che la brevettò nel 1878, e dall'americano Thomas Edison, che ne brevettò un tipo più sofisticato l'anno seguente? Tra i due anglosassoni si scatenò una guerra per la paternità dell'invenzione. Cruto ne restò escluso, eppure nel 1879 realizzò e sperimentò nel laboratorio di fisica dell'Università di Torino un tipo di filamento di carbonio per lampadine che durava 500 ore, contro le 40 di quello di Edison, che dovette spendere altri otto anni per avere un prodotto commerciabile. Anche in questo caso, a Cruto mancavano i soldi per il brevetto e del suo genio si perse memoria.

Non tutti sanno che il personal computer nasce da un' invenzione generata in Olivetti ma sono poi IBM, Hewlett Packard e Apple a svilupparla, a trarne vantaggi e diffonderne l'uso. La P101 (Programma 101) viene realizzata nel 1965 da un gruppo di ricercatori della Olivetti, 
Senza lavoro?

Cambiamento

tecnologico e nuove sfide per

la società

guidati dall'ingegner Piergiorgio Perotto. Chi ne ricorda il nome? Questo computer dalle misure di una macchina da scrivere, pensato per un uso individuale e persino domestico, viene presentato lo stesso anno alla fiera di New York. I calcolatori dell'epoca erano enormi e necessitavano di programmatori esperti per essere utilizzati; il vantaggio della P101 appariva dunque subito evidente. Infatti, la stampa statunitense e altre aziende ne capirono la portata e l'originalità. Gli scienziati della Nasa ne acquistarono 45 per compilare le mappe lunari ed elaborare la traiettoria del viaggio della missione Apollo 11, che nel 1969 portò l'uomo sulla luna. Hewlett Packard ne comprò 100 e lanciò un prodotto del tutto simile. Dovrà poi pagare 900000 dollari all'Olivetti per averne violato il brevetto, ma i vantaggi che ne ha tratto sono stati ben superiori.

Tre soli esempi, che segnalano come la storia italiana veda spesso invenzioni che, per carenza di risorse economiche o per mancanza di adeguate strutture di sostegno, hanno perduto il loro primato e sono state sfruttate economicamente in altri Paesi.

Per contrastare questo rischio è dunque necessario non solo investire in ricerca e svi- 
Franca ALACEVICH

luppo ${ }^{3}$, ma promuovere politiche di sostegno all'innovazione e alla sua valorizzazione sul piano economico, sia attraverso un rapporto più stretto tra università e mondo delle imprese sia operando per sfruttare al meglio le grandi risorse di competenze presenti nelle università e nei centri di ricerca. Si tratta di politiche che richiedono di essere impostate in una strategia di medio-lungo periodo, che contrastano con la ricerca di risultati a breve che detta da anni l'agenda nel Paese, sia nel governo della cosa pubblica sia nel settore privato dell'economia.

A lato di queste politiche, e per affrontare da protagonisti il processo di cambiamento in atto, assume centralità l'investimento nella formazione iniziale e nella formazione continua, che, come si è ricordato, può consentire la riconversione professionale di molti e l'aggiornamento costante di tutti.

3 Anche su questo l'Italia è agli ultimi posti tra i paesi avanzati, nonostante un incremento registrato negli ultimi anni (+20\% dal 2015), specie nella sua componente privata (Visco, Considerazioni finali del Governatore, 2018). 
Senza lavoro?

Cambiamento

tecnologico e nuove sfide per

la società

Non pare, tuttavia, che questa strada sia stata decisamente intrapresa in Italia. Nella Premessa a un recente volume in cui ha raccolto alcuni dei suoi più recenti interventi (Anni difficili, 2018), il Governatore della Banca d'Italia Ignazio Visco segnala come la crisi abbia accentuato problemi che purtroppo già caratterizzavano il paese anche precedentemente:

L'economia italiana si è trovata ad affrontare la crisi finanziaria globale del 2007-08 in condizioni di debolezza strutturale, di stasi della produttività, di carenze infrastrutturali. Le imprese manifestavano un grave ritardo, non limitato all'industria manifatturiera, nell'utilizzo delle nuove tecnologie e nella risposta alla concorrenza dei paesi emergenti [...] Date queste condizioni 'iniziali', gli effetti della crisi finanziaria, pur generata ben al di fuori del nostro paese, hanno finito per essere più gravi da noi che nella maggior parte degli altri paesi avanzati (pp. 7-8).

Infine, è inevitabile ricordare che sono indispensabili anche altre politiche, quelle sociali, rivolte a non abbandonare a se stessi coloro che pagano di più con la perdita del lavoro e del reddito da lavoro. Sono indispensabili an- 
Franca ALACEVICH zitutto per rispetto dei principi democratici su cui si fonda la Costituzione. Tuttavia, lo sono anche per ragioni per così dire strumentali: per mantenere alti i consumi e per ridurre le spese sanitarie e assistenziali, per esempio.

Per concludere, non si può sapere se la nuova rivoluzione tecnologica creerà più lavoro di quanto ne distruggerà, o viceversa. Non lo si può sapere perché ogni stima si basa su dei presupposti che riguardano e prefigurano alcune delle possibili strategie degli attori sociali (imprese, soggetti individuali e collettivi, politica). Ed è da queste strategie che dipende il futuro.

Non è dunque utile, né produttivo, affidarsi a visioni del tutto pessimistiche $o$, al contrario, troppo ottimistiche del futuro del lavoro in relazione ai processi di innovazione tecnologica che stanno prendendo sempre più corpo. Probabilmente, come in passato, gli effetti di tali processi colpiranno di più alcune regioni e alcuni paesi rispetto ad altri, alcune occupazioni rispetto ad altre. Ma le conseguenze per i singoli individui e per la società di questi processi dipenderanno in misura significativa dalla consapevolezza e dalla capacità di sviluppare strategie adeguate.

56 


\section{Bibliografia}

Autor D.H. 2015, Why Are There Still So Many Jobs? The History and Future of Workplace Automation, "Journal of Economic Perspectives», XXIX (3), pp. 3-30.

Bell D. 1973, The Coming of the Post-Industrial Society. A Venture in Social Forecasting, Basic Books, New York.

Brynjolfsson E., McAfee A. 2014, The Second Machine Age. Work, Progress, and Prosperity in a Time of Brilliant Technologies, Norton \& Company, New York (cit. da La nuova rivoluzione delle macchine. Lavoro e prosperità nell'era della tecnologia trionfante, Feltrinelli, Milano 2015).

Deane Ph.M. 1965, The First Industrial Revolution, Cambridge University Press, London (cit. da La prima rivoluzione industriale, il Mulino, Bologna 1990).

Franca Alacevich, Senza lavoro? Cambiamento tecnologico e nuove sfide per la società, (C) 2019 FUP, CC BY 4.0 International, published by Firenze University Press (www.fupress.com),

ISSN 2704-5935 (online), ISBN 978-88-5518-006-1 (online PDF) 
Franca

ALACEVICH

Ford H. 1922, My Life and Work, Garden City New York (ed. it. La mia vita e la mia opera, Apollo, Bologna 1925).

Frey B., Osborne M. 2013, The Future of Employment. How Susceptible are Jobs to Computerization, working paper dell'Oxford Martin School, Università di Oxford, <www. oxfordmartin.ox.ac.uk/downloads/academic/The_Future_of_Employment.pdf $>$ (07/2019), pubblicato anche in «Technological Forecasting \& Social Change», CXIV, 2017, pp. 254-280 (da cui si cita).

Keynes J.M. 1936, The General Theory of Employment, Interest and Money, Macmillan, London (cit. da Teoria generale dell'occupazione, dell'interesse e della moneta, UTET, Torino 1978).

OECD 2018, Education at a Glance 2018: OECD Indicators, OECD Publishing, Paris, $<$ http://dax.doi.org/10.1787/eag-2018-en> (07/2019).

Marx K. 1974, Il Capitale. Libro primo. Capitolo Tredicesimo: Macchine e grande industria (ed. orig. 1867), Editori Riuniti, Roma.

Polanyi M. 1966, The Tacit Dimension, Doubleday, New York.

58 
Senza lavoro?

Cambiamento

tecnologico e nuove sfide per la società

Ricardo D. 1817, On the Principles of Political Economy and Taxation (cit. da Sui principi dell'economia politica e della tassazione, ISEDI, Milano 1976).

Rifkin J. 1995, The End of Work. The Decline of the Global Labor Force and the Dawn of the Post-Market Era, Penguin Putnam, New York (cit. da La fine del lavoro. Il declino della forza lavoro globale e l'avvento dell'era postmercato, Mondadori, Milano 2002).

Smith A. 1776, An Inquiry into the Nature and Causes of the Wealth of Nations (cit. da La Ricchezza delle nazioni, Guida, Napoli 1974). Smith A. 1896, Lectures on Justice, Police, Revenue and Arms (ed. orig. 1763), Clarendon Press, Oxford (cit. da Lezioni di Glasgow, Guida, Napoli 1974).

Taylor F. 1911, The Principles of Scientific Management (ed. it. L'organizzazione scientifica del lavoro, Etas Kompass, Milano 1970).

Tonarelli A. 2018, La Quarta Rivoluzione industriale sarà un'opportunità per le donne?, in A. Cipriani, A. Gramolati, G. Mari (a cura di), Il lavoro 4.0. La quarta rivoluzione industriale e le trasformazioni delle attività lavorative, Firenze University Press, Firenze. 
Franca

ALACEVICH
Visco I. (2018) Anni difficili. Dalla crisi finanziaria alle nuove sfide per l'economia, Bologna, Il Mulino

Visco I. (2018) Considerazioni finali del Governatore, Relazione annuale, Roma, 29 maggio World Economic Forum (2016) The Future of Jobs. Employment, Skills and Workfoce Srategy for the Fourth Industrial Revolution, Global Challenge Insight Report, http://www3.weforum.org/docs/WEF_Future_of_Jobs.pdf. 


\title{
Lectio Magistralis
}

\author{
Titoli pubblicati
}

Sandro Rogari, Nazione e Stato nella storia d'Italia, 2010

Gustavo Zagrebelsky, La difficile democrazia, 2010

Alessandro Cigno, Debito pubblico, ricchezza privata e politiche per la famiglia, 2012

Colin Crouch, Europe and problems of marketization: from Polanyi to Scharpf, 2013

Luigi Dei, Maria Skłodowska Curie: l'ostinata abnegazione di un genio, 2013

Roberto Casalbuoni, La ricerca del bosone di Higgs, 2013

Giusto Puccini, Rendimento e attualità della Costituzione repubblicana, 2014

Marino Regini, La riforma universitaria nel quadro dei sistemi di governance europei, 2014

Sergio Caruso, Per una nuova filosofia della cittadinanza, 2014

Nicoletta Maraschio, La lingua italiana tra passato e futuro, 2015

Carlo Fusaro, Rappresentare e governare: da grande regola a tallone d'Achille del governo parlamentare, 2015

Jürgen Kocka, Capitalism is not democratic and democracy not capitalistic. Tensions and opportunities in historical perspective, 2015

Massimo Morisi, Tra regole ed eccezioni. La messa in opera delle politiche pubbliche in Italia, 2016

Carlo Trigilia, Crescita economica e coesione sociale nelle democrazie avanzate. Un divorzio inevitabile?, 2017

Giuliano Amato, Le identità nazionali nell'Unione Europea, 2018

Gustavo De Santis, Il sistema previdenziale pubblico tra vincoli e scelte, 2019

Marc Lazar, I mutamenti delle democrazie contemporanee. I casi della Francia e dell'Italia, 2019

Michele Casalini, Luigi Dei, Mauro Guerrini, Andrea Zorzi, Conferimento della Laurea magistrale ad honorem in scienze archivistiche e biblioteconomiche a Michele Casalini / Award of the Laurea magistrale ad honorem in Library and information Science to Michele Casalini, 2019

Franca Alacevich, Senza lavoro? Cambiamento tecnologico e nuove sfide per la società, 2019 
